\title{
Pretemporal trans-Meckel's cave transtentorial approach for large petroclival meningiomas
}

\author{
Chih-Hsiang Liao, MD, ${ }^{1}$ Jui-To Wang, MD, ,,4 Chun-Fu Lin, MD, ,,4 Shao-Ching Chen, MD, ${ }^{2}$ \\ Chung-Jung Lin, MD, ,3 Sanford P. C. Hsu, MD, ${ }^{2,4}$ and Min-Hsiung Chen, MD ${ }^{2,4}$ \\ 'Department of Neurosurgery, Neurological Institute, Taichung Veterans General Hospital, Taichung; '2Department of \\ Neurosurgery, Neurological Institute, and ${ }^{3}$ Department of Radiology, Taipei Veterans General Hospital; and ${ }^{4}$ National Yang Ming \\ University, School of Medicine, Taipei, Taiwan
}

OBJECTIVE Despite the advances in skull base techniques, large petroclival meningiomas (PCMs) still pose a challenge to neurosurgeons. The authors' objective of this study was to describe a pretemporal trans-Meckel's cave transtentorial approach for large PCMs and to report the surgical outcomes.

METHODS From 2014 to 2017, patients harboring large PCMs (>3 cm) and undergoing their first resection via this procedure at the authors' institute were included. In combination with pretemporal transcavernous and anterior transpetrosal approaches, the trans-Meckel's cave transtentorial route was created. Surgical details are described and a video demonstrating the procedure is included. Retrospective review of the medical records and imaging studies was performed.

RESULTS A total of 18 patients ( 6 men and 12 women) were included in this study, with mean age of 53 years. The mean sizes of the preoperative and postoperative PCMs were $4.36 \mathrm{~cm} \times 4.09 \mathrm{~cm} \times 4.13 \mathrm{~cm}$ (length $\times$ width $\times$ height) and $0.83 \mathrm{~cm} \times 1.08 \mathrm{~cm} \times 0.75 \mathrm{~cm}$, respectively. Gross-total removal was performed in 7 patients, near-total removal $(>95 \%)$ in 7 patients, and subtotal removal in 4 patients (>90\% in 3 patients and $>85 \%$ in 1 patient). There were no surgical deaths or patients with postoperative hemiplegia. Surgical complications included transient cranial nerve (CN) III palsy (all patients, resolved in 3 months), transient CN VI palsy (2 patients), CN IV palsy (3 patients, partial recovery), hydrocephalus (3 patients), and CSF otorrhea (1 patient). Temporal lobe retraction-related neurological deficits were not observed.

CONCLUSIONS A pretemporal trans-Meckel's cave transtentorial approach offers large surgical exposure and multiple trajectories to the suprasellar, interpeduncular, prepontine, and upper-half clival regions without overt traction, which is mandatory to remove large PCMs. To unlock Meckel's cave where a large PCM lies abutting the cave, pretemporal transcavernous and anterior transpetrosal approaches are prerequisites to create adequate exposure for the final transMeckel's cave step.

https://thejns.org/doi/abs/10.3171/2018.1.FOCUS17733

KEY WORDS anterior transpetrosal; Dolenc-Kawase approach; petroclival meningioma; pretemporal approach; skull base surgery; transcavernous; trans-Meckel's cave transtentorial approach

$\mathrm{P}$ ETROClival meningiomas (PCMs) arise from the petroclival region with significant dural adherence overlying the sphenopetroclival bony interface. They are seated medial to the internal auditory meatus and posterior to the gasserian ganglion, which differentiates them from clival meningiomas that arise close to the midline of the clivus. ${ }^{9}$ Large PCMs always occupy the suprasellar, interpeduncular, prepontine, and clival regions. Despite the advances in skull base techniques designed to minimize morbidity and mortality, large PCMs still pose a great challenge to neurosurgeons. ${ }^{2,9}$

The pretemporal trans-Meckel's cave transtentorial approach is conceptually creating a widened gateway between the middle and posterior fossae, which is performed in combination with pretemporal transcavernous ${ }^{3,6}$ and anterior transpetrosal ${ }^{5}$ approaches (Fig. 1). In this study, the authors describe the pretemporal trans-Meckel's cave transtentorial approach to manage large PCMs, review the

ABBREVIATIONS ACP = anterior clinoid process; $C N=$ cranial nerve; $C S=$ cavernous sinus; $I C A=$ internal carotid artery; $m R S=$ modified Rankin Scale; $P C M=$ petroclival meningioma.

SUBMITTED December 1, 2017. ACCEPTED January 24, 2018.

INCLUDE WHEN CITING DOI: 10.3171/2018.1.FOCUS17733. 


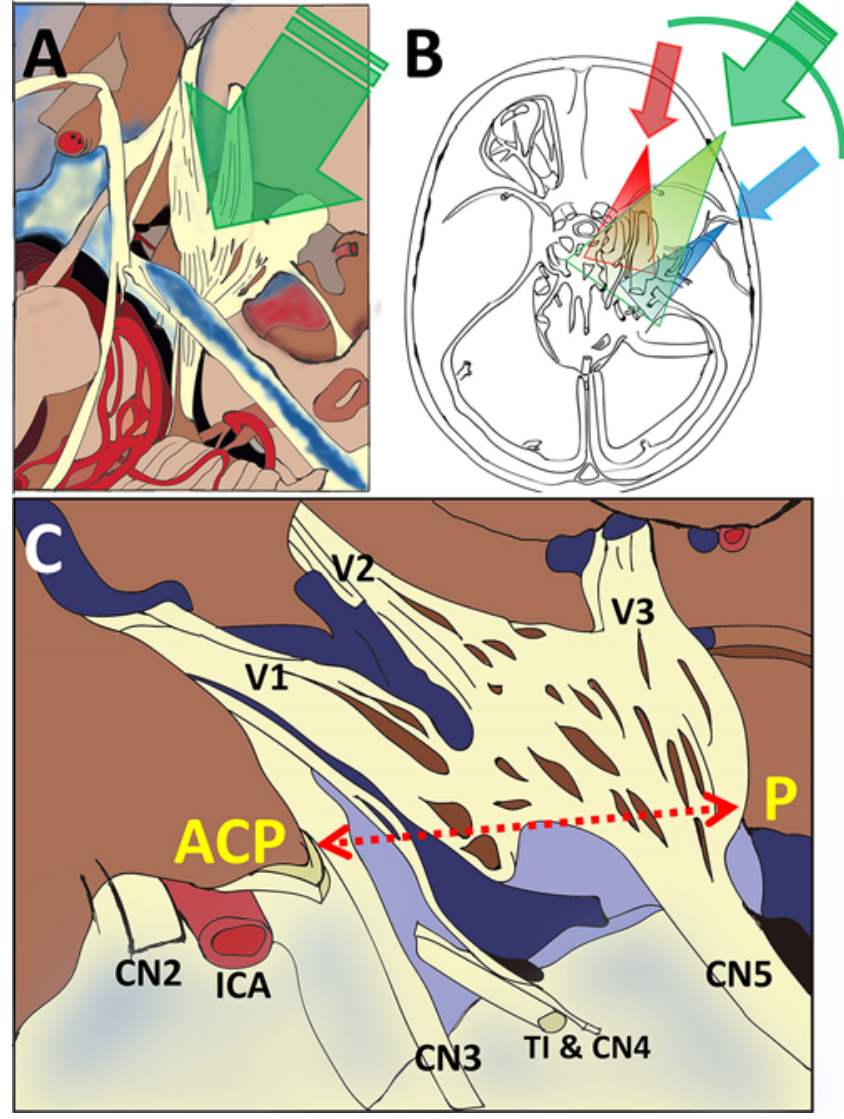

FIG. 1. Illustrations of the pretemporal trans-Meckel's cave transtentorial approach. A: Opening Meckel's cave and the tentorium enlarges the gateway from the middle to the posterior cranial fossa. The arrow indicates the surgical route of this approach. B: The red arrow represents the pretemporal transcavernous approach, the blue arrow represents the anterior transpetrosal approach, and the green arrow indicates the trans-Meckel's cave transtentorial approach. To unlock Meckel's cave to provide multiple trajectories, pretemporal transcavernous and anterior transpetrosal approaches are prerequisites for the final step. C: In this trans-Meckel's cave transtentorial procedure (surgical view), the lateral wall of the CS is peeled to the tentorial incisura ( $\mathrm{TI})$. The dural incision is made from the ACP tip to the petrous apex $(P)$ (dotted line), which frees the temporal lobe. Then, opening the Meckel's cave/tentorium and ligating the superior petrosal sinus provide a more panoramic view to the surgical field. Copyright Sanford P. C. Hsu. Published with permission.

surgical outcomes, and discuss the nuances of this surgical procedure.

\section{Methods}

This was a retrospective study. Since 2014, our senior author (S.P.C.H.) has routinely used the pretemporal trans-Meckel's cave transtentorial approach for large PCMs. From January 2014 to August 2017, a total of 93 patients harboring skull base meningiomas at the middle/ posterior fossa and operated on by the senior author were reviewed. Patients with large PCMs $(>3.0 \mathrm{~cm} \times 3.0 \mathrm{~cm}$ $\times 3.0 \mathrm{~cm}$ in length, width, and height) undergoing their first resection via this approach were included. PCMs less than $3 \mathrm{~cm}$ in any of the 3 dimensions were excluded. Cavernous sinus (CS), petrous, clival, and cerebellopontine angle meningiomas with petroclival extension were also excluded. Surgical procedures are described below, and shown in Video 1.

VIDEO 1. This video clip demonstrates a case of a right-sided giant PCM, approximately $5.0 \mathrm{~cm} \times 5.0 \mathrm{~cm} \times 5.0 \mathrm{~cm}$, causing symptoms of unsteady gait, dysphagia, and dysarthria. The patient underwent a pretemporal trans-Meckel's cave transtentorial approach. The tumor was gross-totally removed. There was transient diplopia and ptosis after the operation. The pathological study proved the PCM to be a transitional type meningioma. No tumor recurrence was noted in the last MRI follow-up. Copyright Sanford P. C. Hsu. Published with permission. Click here to view.

Retrospective review of the medical records and imaging studies was performed.

\section{Surgical Procedures}

\section{Patient Position and Skin Flap Design}

Under general anesthesia and intraoperative neuromonitoring, the patient is positioned supine with the head fixed by headpins and the head holder, elevated above the level of the heart, extended and rotated $30^{\circ}$ to the opposite side to bring the malar eminence to the highest point of the operative field. A curvilinear frontotemporal incision begins at the superior border of the zygomatic arch, close to the tragal cartilage and anterior to the superficial temporal artery. The incision then proceeds superiorly and curves anteriorly to the frontal region behind the hairline. Interfascial dissection is applied to preserve the frontotemporal branch of the facial nerve. ${ }^{13}$ The skin flap is reflexed down and hooked to expose the upper margin of the zygomatic arch. Two beveled cuts are made by electric drills at the anterior and posterior ends of the zygomatic arch, to make the fractured zygoma a reversed trapezoid shape (Fig. 2). This step helps to achieve inferior reflection of the temporalis muscle. No screws and plates are needed when we place the zygoma back due to its reversed trapezoid shape at the end of the operation. Leaving a muscle cuff on the superior temporal line for later suturing, the temporalis is reflected down along with the fractured zygoma.

\section{Craniotomy}

The craniotomy used in this procedure is a standard pterional craniotomy with temporal extension. After the craniotomy flap is established, the temporal squama is drilled in its anterolateral extension flush with the floor of the middle fossa. The sphenoid wing is then drilled from its lateral to medial extension until the anterior clinoid process $(\mathrm{ACP})$ is reached. This exposes the meningio-orbital artery, which is coagulated and cut. During this step, the posterior third of the lateral and superior orbital wall is removed, preserving the periorbita. The ACP is thus exposed and removed as described below. The craniotomy defects at the end of the operation are repaired using bone replacement materials.

\section{Epidural Dissection of the Lateral Wall of the CS, ACP Removal, and Anterior Petrosectomy}

The epidural dissection of the lateral wall of the CS, and ACP removal have been described previously. ${ }^{3,6,7} \mathrm{~A}$ few points are mentioned here. The meningio-orbital artery is a point where the dissection plane between the dura 

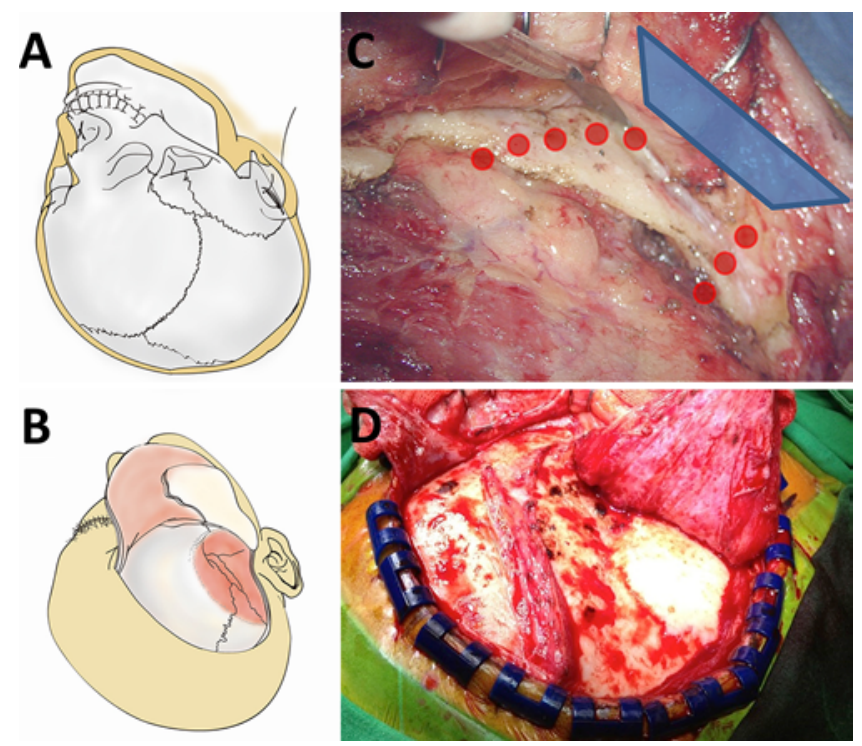

FIG. 2. Patient positioning and flap design. A: The patient's head is elevated above the level of the heart, extended and rotated $30^{\circ}$ to the opposite side to bring the malar eminence to the highest point of the operative field. B: A curvilinear frontotemporal incision begins at the superior border of the zygomatic arch, close to the tragal cartilage and anterior to the superficial temporal artery, to the frontal region behind the hairline. C: Two beveled cuts are made by electric drills at the anterior and posterior ends of the zygomatic arch (red dots), to make the fractured zygoma a reversed trapezoid shape (blue trapezoid). No screws/plates are needed when placing the zygoma back due to its shape. D: Interfascial dissection is applied to preserve the frontotemporal branch of the facial nerve. Leaving a muscle cuff on the superior temporal line for later suturing, the temporalis is reflected down along with the fractured zygoma. Copyright Sanford P. C. Hsu (panels A and B). Published with permission.

propria of the temporal lobe and the lateral wall of the CS starts, and cranial nerves (CNs) III and IV, and V1 and $\mathrm{V} 2$ nerves are identified. The space between the V1 and $\mathrm{V} 2$ branches is a common area for bleeding that can be controlled by fibrin glue injection. After peeling the lateral wall of the CS, the ACP removal can be performed. Drilling should be directed over the superior and lateral aspects of the optic canal first with copious irrigation, which does not extend medially over the optic nerve to prevent risks of entering the spheno-ethmoidal air cells with subsequent CSF rhinorrhea.

An anterior transpetrosal approach ${ }^{5}$ can be accomplished either before or after the ACP removal. We continue the extradural dissection to expose V3, ligate the middle meningeal artery, and identify the greater superficial petrosal nerve. During anterior petrosectomy, a vascular Doppler system is used to identify the location of the petrous internal carotid artery (ICA).

\section{Trans-Meckel's Cave Transtentorial Approach}

Opening Meckel's cave and the tentorium are paramount in removing large PCMs. Via interdural dissection, we expose the gasserian ganglion and peel the lateral wall of the CS to the tentorial incisura. We use multiple tack-up sutures on the dura over the temporal lobe to maintain the surgical field and to protect the vein of Labbé. After fin- ishing the extradural work, we cut open the dura linearly, following the sphenoid wing indentation, all the way down to the ACP tip that has been removed extradurally. From the ACP tip, the durotomy extends toward the frontal side, mobilizing the ICA and CN II by cutting the distal dural ring and the falciform ligament, respectively; the other cut extends toward the temporal side, along the tentorial incisura to the anterior petrous region where the anterior petrosectomy has been performed. Then, we open Meckel's cave over the gasserian ganglion toward the tentorial incisura (Fig. 1). The trigeminal nerve can be mobilized to facilitate more bone removal at the petrous apex where the lateral limit is the internal acoustic canal and the medial limit is Dorello's canal. Tumor debulking is performed. The tentorium is coagulated and cut along with the tumor. The superior petrosal sinus is ligated and dissected to create more space. CNs IV and VI should be preserved during tumor removal.

\section{Wound Closure}

The dura is closed by running suture, and the dural defect at the skull base is repaired with artificial dura, fat graft, and fibrin glues. The bone flap is placed back and augmented by bone replacement materials. The temporalis muscle is sutured back to the muscle cuff left on the bone flap, and the fractured trapezoid-shaped zygoma is embedded back in place without the need of screws and plates. The skin flap is then closed in standard fashion.

\section{Results}

\section{Age and Sex}

Eighteen patients harboring large PCMs were included in this series. There were 6 men and 12 women, whose ages ranged from 31 to 75 years (mean 53.3 years).

\section{Clinical Presentation}

Eleven patients $(61 \%)$ presented with unsteady gait/ limb weakness; 5 (28\%) with swallowing difficulty/choking; 5 (28\%) with facial pain/dysesthesia/numbness; 4 (22\%) complained of hearing impairment; and 3 patients $(17 \%)$ had double vision. According to the modified Rankin Scale (mRS), there were 13 patients with an mRS score of 1, 4 with an mRS score of 2, and 1 patient with an $\mathrm{mRS}$ score of 3 .

\section{Imaging Findings and Surgical Outcome}

The mean preoperative size of the PCMs was $4.36 \mathrm{~cm}$ $\times 4.09 \mathrm{~cm} \times 4.13 \mathrm{~cm}$ (length $\times$ width $\times$ height $)$. All of the patients had severe brainstem compression, and basilar artery encasement was observed in 4 patients $(22 \%)$. The postoperative mean size of the PCMs was $0.83 \mathrm{~cm} \times$ $1.08 \mathrm{~cm} \times 0.75 \mathrm{~cm}($ length $\times$ width $\times$ height $)$. Gross-total removal was completed in 7 patients, near-total removal $(>95 \%)$ in 7 patients, and subtotal removal in 4 patients ( $>90 \%$ in 3 patients and $>85 \%$ in 1 patient; Figs. $3-5$ ). Pathologic study showed 9 transitional types, 7 secretory types, 1 microcystic type, and 1 clear cell type. Five patients received Gamma Knife radiosurgery for residual tumor, and 3 patients needed a ventriculoperitoneal shunt 

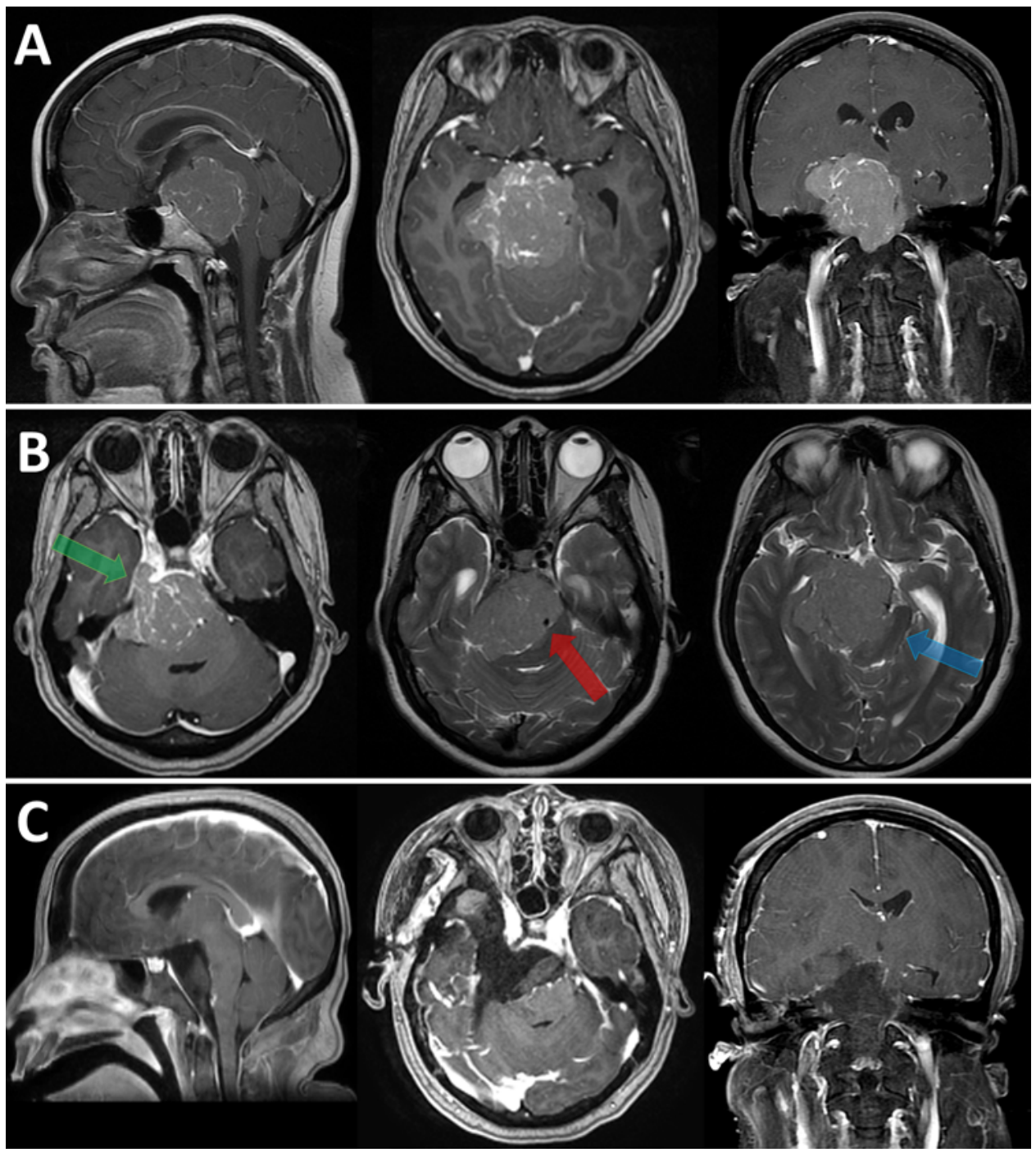

FIG. 3. Gross-total removal of a giant PCM operated via the trans-Meckel's cave approach. A: The preoperative MRI study with contrast administration showed a right-sided giant PCM, about $5.0 \times 5.0 \times 5.0 \mathrm{~cm}^{3}$. The tumor bulged upward to the third ventricle floor, forward to the pituitary stalk and optic apparatus, and backward to the brainstem. B: The green arrow indicates the tumor invading Meckel's cave, the red arrow represents the basilar artery encasement, and the blue arrow indicates the compressed brainstem. C: The postoperative contrasted MRI study showed gross-total removal of the tumor (see Video 1).

due to hydrocephalus. The tumor sizes remained stable in all cases with residual tumor. At the latest follow-up, 14 patients had an mRS score of 0 or 1,3 patients had an $\mathrm{mRS}$ score of 2 , and 1 patient had an mRS score of 3 . The mean duration of follow-up was 18 months (range 2-41 months).

\section{Complications}

There were no surgical deaths or patients with postoperative hemiplegia. Procedure-related transient $\mathrm{CN}$ III palsy occurred in all patients and resolved within 3 months after the operation. Two patients had transient $\mathrm{CN}$ VI palsy. Three patients had CN IV palsy. One patient with postoperative CSF otorrhea needed repair surgery. Temporal lobe retraction-related neurological deficits were not observed.

\section{Discussion}

Large PCMs that arise from the petroclival region with significant dural adherence overlying the spheno-petroclival bone interface always occupy the suprasellar, interpeduncular, prepontine, and clival spaces as well. To remove such tumors involving the middle and posterior fossae, adequate surgical exposure and multiple trajectories without overt retraction of the cerebral parenchyma are important. A pretemporal trans-Meckel's cave transtentorial approach provides sufficient anterior-to-posterior, medial-to-lateral, and superior-to-inferior (and vice versa) trajectories to remove large PCMs (Fig. 1B). To avoid overt brain retraction, multiple tack-up sutures are used to hold the temporal lobe and maintain the surgical field. The whole approach consists of 1) a pretemporal transcavern- 

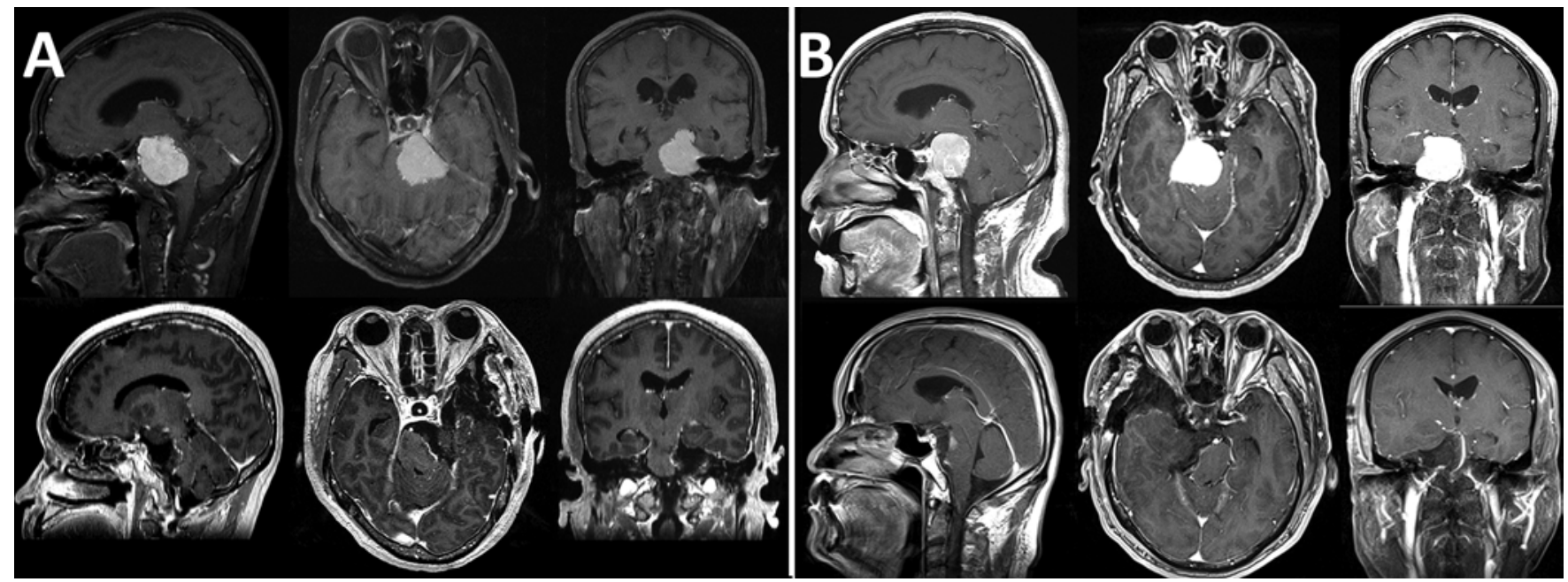

FIG. 4. Gross-total removal of 2 cases of large PCMs operated via the trans-Meckel's cave approach, demonstrated by preoperative contrasted MR images (upper row) and postoperative follow-up images (lower row). A: A left-sided PCM, approximately $3.5 \times$ $3.4 \times 3.6 \mathrm{~cm}^{3}$. B: A right-sided PCM, approximately $3.1 \times 4.0 \times 3.5 \mathrm{~cm}^{3}$.

ous approach, 3,6,7 2) an anterior transpetrosal approach, $, 5,12$ and, finally, 3) the trans-Meckel's cave transtentorial step.

Judicious bone removal and mobilization of neurovascular structures is the key for skull base approaches. To unlock Meckel's cave where a large PCM lies abutting the cave, pretemporal transcavernous and anterior transpetrosal approaches are prerequisites to create adequate exposure for the final step. With a pretemporal transcavernous approach, the surgical corridor can reach suprasellar, interpeduncular, and upper-third clival regions. We
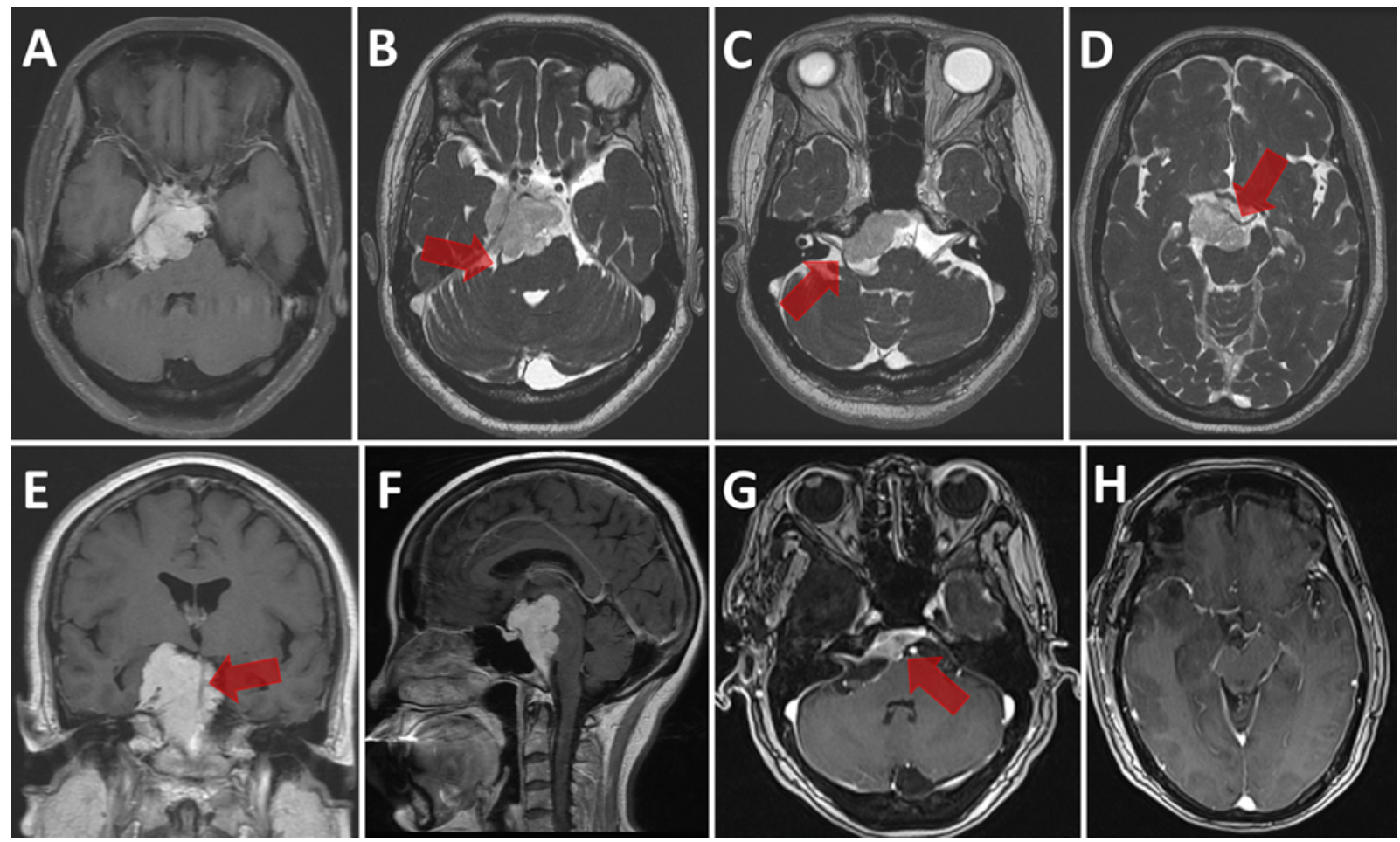

FIG. 5. Subtotal tumor removal of a large PCM operated via the trans-Meckel's cave approach. A and B: MRI study showed a right-sided large PCM, about $3.8 \times 3.6 \times 5.0 \mathrm{~cm}^{3}$, compressing the brainstem. The arrow indicates CN V. C: The lower part of the tumor compressed the nerve root entry zone of CNs VII and VIII (arrow). D: The upper part of the tumor compressed the cerebral peduncle and encased the posterior cerebral artery (arrow). E and F: The tumor encased the basilar artery (arrow) and extended down to the lower clivus. G: The residual tumor hid behind the lower clivus (arrow), which showed the limit of this approach. $\mathrm{H}$ : The interpeduncular part of the tumor was completely removed. 
mobilize the ICA and CNs II-IV to avoid neurovascular injury. The basilar artery can be traced from the posterior communicating artery during tumor removal. With an anterior transpetrosal approach, removing the anterior petrous apex provides a gateway to the posterior cranial fossa to remove tumor parts situated medial to the internal acoustic meatus. In the final trans-Meckel's cave transtentorial step, the lateral wall of the CS is peeled to the tentorial incisura, and the dural incision is made from the ACP tip to the petrous apex, which frees the temporal lobe (Fig. 1C). Then, by multiple tack-up sutures, the temporal lobe can be held in place. The gasserian ganglion is exposed and mobilized to help further anterior petrous apex removal, which creates more space in the medial-to-lateral trajectory to the prepontine/midclival region and posterior fossa. During tumor removal, we have to manage to find the petroclival ligament/Dorello's canal for CN VI medial to Meckel's cave. The brainstem can be exposed by tracing the trigeminal nerve to the nerve root entry zone.

In the literature, the mean length of the clivus in adults was reported as $45 \mathrm{~mm} .{ }^{8}$ Quantitative studies showed that the transcavernous approach exposed lesions located 6.0 $\mathrm{mm}$ below the floor of the sella, ${ }^{4}$ and the anterior transpetrosal approach extending up to $18 \mathrm{~mm},{ }^{1}$ although the transcavernous approach offers more advantages than the anterior transpetrosal approach for retrosellar lesions. ${ }^{4}$ The traditional anterior transpetrosal (Kawase) approach provides a $10 \times 5 \mathrm{~mm}$ fenestration at the petrous apex of the temporal bone between $\mathrm{CN} \mathrm{V}$ and the internal acoustic canal. By mobilizing the mandibular branch of the trigeminal nerve medially, Tripathi et al. proposed a modified Dolenc-Kawase approach to create a 1.5-times larger area at the anterior petrous apex compared with the traditional Kawase approach. ${ }^{12}$ However, their so-called modified Dolenc-Kawase approach did not include ACP removal and ICA/optic nerve mobilization. In our series, we performed a true combination of the pretemporal transcavernous and anterior transpetrosal approaches and extended their limits by a trans-Meckel's cave transtentorial procedure, which is suitable for large to giant PCMs.

\section{Outcomes and Complications}

There were no deaths or patients with hemiplegia in this series. No neurological deterioration due to brain retraction or injury to the vein of Labbé complex was found. Procedure-related complications were transient CN III and VI palsies, which resolved 3 months after the operation. We pursued gross-total removal of the tumor, but did not require it in every patient due to the possibility of neurological deficits. We left a piece of tumor if severe adhesion at the brainstem-tumor interface or perforator encasement of the basilar artery was noted. Stereotactic radiosurgery affords a reasonable tumor control rate for skull base meningiomas less than $14 \mathrm{~cm}, 3,10,11$ which helps to address the residual tumor after the open surgery.

\section{Limitations of the Study}

Although the exposure and trajectories obtained through the trans-Meckel's cave approach is greater, the lower clival region is still difficult to reach because of an inherent anatomical barrier (Fig. 5). The major factors of tumor resection and preservation of critical structures are determined by tumor consistency, arachnoid plane preservation, and degree of involvement of critical neurovascular structures. ${ }^{2}$ Although this is a small retrospective study, the contribution of our series is in describing the surgical concept about the new gateway (Meckel's cave) between the middle and posterior fossae with an entirely extradural approach that provides enhanced exposure and multiple trajectories. The temporal lobe and vein of Labbé are protected by the dura peeled from the lateral dural wall of the CS all the way to the tentorial incisura. Tailored extradural modifications can be applied for middle cranial fossa lesions with variable extension into the anterior and posterior fossae.

\section{Conclusions}

A pretemporal trans-Meckel's cave transtentorial approach offers larger surgical exposure and multiple trajectories to the suprasellar, interpeduncular, prepontine, and upper-half clival regions without overt brain retraction, which is mandatory to remove large PCMs. To unlock Meckel's cave where a large PCM lies abutting the cave, pretemporal transcavernous and anterior transpetrosal approaches are prerequisites to create adequate exposure for the final step.

\section{Acknowledgments}

We thank Hui-Ching Ho, medical illustrator in the Teaching Material Support Group, Center for Faculty Development, Taichung Veterans General Hospital, for her artwork assistance in the figures and Video 1. One anatomical illustration used in Video 1 (at the 01:50-01:57 mark) is attributed to the Rhoton Collection (rhoton.ineurodb.org), and we have complied with the terms of use of the Rhoton collection (CC BY-NC-SA 4.0 license; https:// creativecommons.org/licenses/by-nc-sa/4.0/).

\section{References}

1. Aziz KM, Tew JM Jr, Chicoine MR: The Kawase approach to retrosellar and upper clival basilar aneurysms. Neurosurgery 44:1225-1236, 1999

2. Bambakidis NC, Kakarla UK, Kim LJ, Nakaji P, Porter RW, Daspit CP, et al: Evolution of surgical approaches in the treatment of petroclival meningiomas: a retrospective review.

Neurosurgery 62 (6 Suppl 3):1182-1191, 2008

3. Dolenc VV: A combined epi- and subdural direct approach to carotid-ophthalmic artery aneurysms. J Neurosurg 62:667672,1985

4. Figueiredo EG, Zabramski JM, Deshmukh P, Crawford NR, Spetzler RF, Preul MC: Comparative analysis of anterior petrosectomy and transcavernous approaches to retrosellar and upper clival basilar artery aneurysms. Neurosurgery 58 (1 Suppl):ONS13-ONS21, 2006

5. Kawase T, Shiobara R, Toya S: Anterior transpetrosaltranstentorial approach for sphenopetroclival meningiomas: surgical method and results in 10 patients. Neurosurgery 28:869-876, 1991

6. Krisht AF: Transcavernous approach to diseases of the anterior upper third of the posterior fossa. Neurosurg Focus 19(2):E2, 2005

7. Krisht AF, Kadri PA: Surgical clipping of complex basilar apex aneurysms: a strategy for successful outcome using the pretemporal transzygomatic transcavernous approach. Neurosurgery 56 (2 Suppl):261-273, 2005

8. Lang J, Issing P: [The measurements of the clivus, the foram- 
ina on the external base of the skull and the superior vertebrae.] Anat Anz 169:7-34, 1989 (Ger)

9. Maurer AJ, Safavi-Abbasi S, Cheema AA, Glenn CA, Sughrue ME: Management of petroclival meningiomas: a review of the development of current therapy. J Neurol Surg B Skull Base 75:358-367, 2014

10. Starke RM, Przybylowski CJ, Sugoto M, Fezeu F, Awad AJ, Ding D, et al: Gamma Knife radiosurgery of large skull base meningiomas. J Neurosurg 122:363-372, 2015

11. Starke RM, Williams BJ, Hiles C, Nguyen JH, Elsharkawy MY, Sheehan JP: Gamma Knife surgery for skull base meningiomas. J Neurosurg 116:588-597, 2012

12. Tripathi M, Deo RC, Suri A, Srivastav V, Baby B, Kumar S, et al: Quantitative analysis of the Kawase versus the modified Dolenc-Kawase approach for middle cranial fossa lesions with variable anteroposterior extension. J Neurosurg 123:14-22, 2015

13. Yaşargil MG, Reichman MV, Kubik S: Preservation of the frontotemporal branch of the facial nerve using the interfascial temporalis flap for pterional craniotomy. Technical article. J Neurosurg 67:463-466, 1987

\section{Disclosures}

The authors report no conflict of interest concerning the materi- als or methods used in this study or the findings specified in this paper.

\section{Author Contributions}

Conception and design: Hsu, Liao. Acquisition of data: Liao, Wang, SC Chen, CJ Lin. Analysis and interpretation of data: Liao, Wang, CF Lin, SC Chen, CJ Lin. Drafting the article: Liao, Wang, CF Lin, SC Chen, CJ Lin. Critically revising the article: Hsu, Liao, CF Lin, SC Chen, CJ Lin, MH Chen. Reviewed submitted version of manuscript: Hsu, Liao, CF Lin, MH Chen. Approved the final version of the manuscript on behalf of all authors: Hsu. Administrative/technical/material support: Hsu, Liao. Study supervision: Hsu, MH Chen.

\section{Supplemental Information \\ Videos}

Video 1. https://vimeo.com/253599600.

Video abstract. https://vimeo.com/258096263.

\section{Correspondence}

Sanford P. C. Hsu: Neurological Institute, Taipei Veterans General Hospital, Taipei, Taiwan.doc3339b@gmail.com. 